\title{
Generalizations of Implication Algebras
}

\author{
By \\ Ivan Chajda ${ }^{1}$ and Helmut Länger \\ (Vorgelegt in der Sitzung der math.-nat. Klasse am 11. Oktober 2007 \\ durch das w. M. Ludwig Reich)
}

\begin{abstract}
Implication algebras, originally introduced in order to study algebraic properties of the implication operation in Boolean algebras, are generalized and it is shown that these more general algebras are in one-to-one correspondence to semilattices with 1 the principal filters of which are posets with an antitone involution, respectively to commutative directoids with 1 the principal filters of which are posets with a switching involution.
\end{abstract}

Mathematics Subject Classification (2000): 20N02, 06A12, 06E05, 06C15.

Key words: Implication algebra, orthoimplication algebra, orthomodular implication algebra, strong $I$-algebra, weak $I$-algebra, semilattice, directoid, Boolean algebra, orthomodular lattice, poset, antitone involution, switching involution.

In order to study algebraic properties of the implication operation in Boolean algebras J. C. AвBOTT introduced the notion of an implication algebra (cf. [1]). He showed that these algebras are in one-toone correspondence to join-semilattices with 1 the principal filters of which are Boolean algebras. These algebras were generalized, e.g. in [2], [8] and [9], where corresponding results were achieved.

Let us mention that also other types of implication in non-classical logic were treated in the literature (see e.g. [4]-[7]). However, these

\footnotetext{
${ }^{1}$ Support of the research of the first author by the Czech Government Research Project MSM 6198959214 is gratefully acknowledged.
} 
can be unified by a more generalized approach which will be presented here.

The aim of this paper is to further generalize the concept of an implication algebra to algebras satisfying weaker conditions. It turns out that by the constructions originally used by J. C. AвBOTT these more general algebras are in one-to-one correspondence to semilattices with 1 the principal filters of which are posets with an antitone involution, respectively to directoids with 1 the principal filters of which are posets with a switching involution.

\section{Implication Algebras, Orthoimplication Algebras and Orthomodular Implication Algebras}

We start our investigations by repeating the definition of an implication algebra and its connection to certain join-semilattices having an additional structure.

Definition 1.1. Let $\mathcal{A}=(A, \cdot, 1)$ be an algebra of type $(2,0)$. $\mathcal{A}$ is called an implication algebra (cf. [1]) if it satisfies

$$
\begin{aligned}
x x & =1, \\
(x y) x & =x, \\
(x y) y & =(y x) x
\end{aligned}
$$

and

$$
x(y z)=y(x z) .
$$

Remark 1.2. The nullary operation 1 can be dropped from the family of fundamental operations of an implication algebra since due to the first identity in the definition it is an algebraic constant.

The following theorem was proved in [1]:

Theorem 1.3. Let $\mathcal{A}=(A, \cdot, 1)$ be an implication algebra. Define

$$
x \vee y:=(x y) y \quad \text { and } \quad x^{y}:=x y
$$

for all $x, y \in A$. Then $\mathbf{S}(\mathcal{A}):=\left(A, \vee,\left({ }^{x} ; x \in A\right), 1\right)$ is an algebra such that $(A, \vee, 1)$ is a join-semilattice with greatest element 1 and for every $x \in A\left([x, 1], \leq,^{x}\right)$ is a Boolean algebra. Conversely, let $\mathcal{S}:=\left(S, \vee,\left({ }^{x} ; x \in S\right), 1\right)$ be an algebra such that $(S, \vee, 1)$ is a joinsemilattice with greatest element 1 and for every $x \in S\left([x, 1], \leq,{ }^{x}\right)$ is a Boolean algebra. Define

$$
x y:=(x \vee y)^{y}
$$


for all $x, y \in S$. Then $\mathbf{A}(\mathcal{S}):=(S, \cdot, 1)$ is an implication algebra. Moreover, $\mathbf{A}(\mathbf{S}(\mathcal{A}))=\mathcal{A}$ and $\mathbf{S}(\mathbf{A}(\mathcal{S}))=\mathcal{S}$ for every implication algebra $\mathcal{A}$ and every algebra $\left.\mathcal{S}=\left(S, \vee,{ }^{x} ; x \in S\right), 1\right)$ such that $(S, \vee, 1)$ is a join-semilattice with greatest element 1 and for every $x \in S$ $\left([x, 1], \leq{ }^{x}\right)$ is a Boolean algebra.

The notion of an implication algebra was generalized to the notion of an orthoimplication algebra, respectively orthomodular implication algebra, as follows:

Definition 1.4. Let $\mathcal{A}=(A, \cdot, 1)$ be an algebra of type $(2,0)$. $\mathcal{A}$ is called an orthoimplication algebra (cf. [2]) if it satisfies

$$
\begin{aligned}
x x & =1, \\
(x y) x & =x, \\
(x y) y & =(y x) x
\end{aligned}
$$

and

$$
x((y x) z)=x z .
$$

Definition 1.5. Let $\mathcal{A}=(A, \cdot, 1)$ be an algebra of type $(2,0)$. $\mathcal{A}$ is called an orthomodular implication algebra (cf. [8] and [9]) if it satisfies

$$
\begin{aligned}
x x & =1, \\
(x y) x & =x, \\
(x y) y & =(y x) x, \\
(((x y) y) z)(x z) & =1
\end{aligned}
$$

and

$$
((((((x y) y) z) x) x) z) x) x=(((x y) y) z) z .
$$

In [2], [8] and [9] it was proved that orthoimplication algebras, respectively orthomodular implication algebras, correspond to joinsemilattices with 1 the principal filters of which are orthomodular lattices satisfying the compatibility condition $(x \leq y \leq z$ implies $z^{y}=z^{x} \vee y$ ) respectively to join-semilattices with 1 the principal filters of which are orthomodular lattices. These correspondences are one-to-one and completely analogous to that proved by J. C. ABBOTT in [1] for implication algebras.

\section{I-Algebras}

We now define a new more general type of implication algebras: 
Definition 2.1. Let $\mathcal{A}=(A, \cdot, 1)$ be an algebra of type $(2,0)$. $\mathcal{A}$ is called a strong I-algebra if it satisfies

$$
\begin{aligned}
1 x & =x, \\
x x & =1, \\
x(y x) & =1, \\
(x y) y & =(y x) x
\end{aligned}
$$

and

$$
(((x y) y) z)(x z)=1 .
$$

Remark 2.2. The class of all strong $I$-algebras forms a variety.

For the following theorem we need the definition of an antitone involution of a poset.

Definition 2.3. Let $(P, \leq)$ be a poset and $f: P \rightarrow P$. $f$ is called an antitone involution of $(P, \leq)$ if $f(x) \geq f(y)$ whenever both $x, y \in P$ and $x \leq y$ and if $f(f(x))=x$ for all $x \in P$.

Now the following result can be proved:

Theorem 2.4. Let $\mathcal{A}=(A, \cdot, 1)$ be a strong I-algebra. Define

$$
x \vee y:=(x y) y \quad \text { and } \quad x^{y}:=x y
$$

for all $x, y \in A$. Then $\mathbf{S}(\mathcal{A}):=\left(A, \vee,\left({ }^{x} ; x \in A\right), 1\right)$ is an algebra such that $(A, \vee, 1)$ is a join-semilattice with greatest element 1 and for every $x \in A\left([x, 1], \leq,^{x}\right)$ is a poset with an antitone involution where $\leq$ denotes the partial order induced by $\vee$. Conversely, let $\mathcal{S}:=\left(S, \vee,\left({ }^{x} ; x \in S\right), 1\right)$ be an algebra such that $(S, \vee, 1)$ is a joinsemilattice with greatest element 1 and for every $x \in S\left([x, 1], \leq,^{x}\right)$ is a poset with an antitone involution. Define

$$
x y:=(x \vee y)^{y}
$$

for all $x, y \in S$. Then $\mathbf{A}(\mathcal{S}):=(S, \cdot, 1)$ is a strong I-algebra. Moreover, $\mathbf{A}(\mathbf{S}(\mathcal{A}))=\mathcal{A}$ and $\mathbf{S}(\mathbf{A}(\mathcal{S}))=\mathcal{S}$ for every strong I-algebra $\mathcal{A}$ and every algebra $\mathcal{S}=\left(S, \vee,\left({ }^{x} ; x \in S\right), 1\right)$ such that $(S, \vee, 1)$ is a joinsemilattice with greatest element 1 and for every $x \in S\left([x, 1], \leq,{ }^{x}\right)$ is a poset with an antitone involution.

Proof. Assume $(A, \cdot, 1)$ to be a strong $I$-algebra and for all $x, y \in A$ define $x \leq y$ if $x y=1, x \vee y:=(x y) y$ and $x^{y}:=x y$. Because of (S2), $\leq$ is reflexive. If $a \leq b$ and $b \leq a$ then

$$
a=1 a=(b a) a=(a b) b=1 b=b
$$


according to (S1) and (S4) proving antisymmetry of $\leq$. If $a \leq b \leq c$ then

$$
a c=1(a c)=(b c)(a c)=((1 b) c)(a c)=(((a b) b) c)(a c)=1
$$

according to (S1) and (S5), i.e., $a \leq c$. This shows transitivity of $\leq$. Hence $(A, \leq)$ is a poset and according to (S2) and (S3), $a 1=$ $a(a a)=1$, i.e., $a \leq 1$ which means that 1 is the greatest element of $(A, \leq)$. According to (S4), $\vee$ is commutative. Because of (S1), (S2) and (S5)

$a(a \vee b)=a((a b) b)=1(a((a b) b))=(((a b) b)((a b) b))(a((a b) b))=1$,

i.e., $a \leq a \vee b$. Hence $b \leq b \vee a=a \vee b$. Because of (S1) and (S5), $a \leq b$ implies

$$
(b c)(a c)=((1 b) c)(a c)=(((a b) b) c)(a c)=1,
$$

i.e., $b c \leq a c$. Therefore $a, b \leq c$ implies $c b \leq a b$ and hence

$$
a \vee b=(a b) b \leq(c b) b=(b c) c=1 c=c
$$

according to (S4) and (S1). Hence $a \vee b$ is the supremum of $a$ and $b$ with respect to $\leq$. If $b \in[a, 1]$ then $a b^{a}=a(b a)=1$ according to (S3), i.e., $b^{a} \in[a, 1]$. Hence ${ }^{a}$ is a unary operation on $[a, 1]$. If $a \leq b \leq c$ then $c^{a}=c a \leq b a=b^{a}$, i.e., ${ }^{a}$ is antitone. If $a \leq b$ then $\left(b^{a}\right)^{a}=(b a) a=(a b) b=1 b=b$ according to (S4) and (S1) and hence $^{a}$ is an involution. Therefore $\left(A, \vee,\left({ }^{a} ; a \in A\right), 1\right)$ is an algebra such that $(A, \vee, 1)$ is a join-semilattice with greatest element 1 and for every $x \in A\left([x, 1], \leq,{ }^{x}\right)$ is a poset with an antitone involution with respect to the partial order induced by $\vee$. Moreover, $((a b) b) b=a b \vee b=a b$ since $b \leq a b$ according to $b(a b)=1$ by (S3).

Conversely, assume $\left(A, \vee,\left({ }^{a} ; a \in A\right), 1\right)$ to be an algebra such that $(A, \vee, 1)$ is a join-semilattice with greatest element 1 and for every $x \in A\left([x, 1], \leq,^{x}\right)$ is a poset with an antitone involution. Moreover, for all $x, y \in A$ define $x y:=(x \vee y)^{y}$.

$$
\begin{aligned}
1 a & =(1 \vee a)^{a}=1^{a}=a, \\
a a & =(a \vee a)^{a}=a^{a}=1, \\
a(b a) & =\left(a \vee(b \vee a)^{a}\right)^{(b \vee a)^{a}}=\left((b \vee a)^{a}\right)^{(b \vee a)^{a}}=1, \\
(a b) b & =\left((a \vee b)^{b} \vee b\right)^{b}=\left((a \vee b)^{b}\right)^{b}=a \vee b=b \vee a \\
& =\left((b \vee a)^{a}\right)^{a}=\left((b \vee a)^{a} \vee a\right)^{a}=(b a) a,
\end{aligned}
$$




$$
\begin{aligned}
(((a b) b) c)(a c) & =\left(\left(\left((a \vee b)^{b} \vee b\right)^{b} \vee c\right)^{c} \vee(a \vee c)^{c}\right)^{(a \vee c)^{c}} \\
& =\left(\left(\left((a \vee b)^{b}\right)^{b} \vee c\right)^{c} \vee(a \vee c)^{c}\right)^{(a \vee c)^{c}} \\
& =\left((a \vee b \vee c)^{c} \vee(a \vee c)^{c}\right)^{(a \vee c)^{c}} \\
& =\left((a \vee c)^{c}\right)^{(a \vee c)^{c}}=1
\end{aligned}
$$

Therefore $(A, \cdot, 1)$ is a strong $I$-algebra. Moreover, $\left((a \vee b)^{b} \vee b\right)^{b}=$ $\left((a \vee b)^{b}\right)^{b}=a \vee b$ and if $a \leq b$ then $(b \vee a)^{a}=b^{a}$.

Next we define a generalization of the notion of a strong $I$-algebra: Definition 2.5. Let $\mathcal{A}=(A, \cdot, 1)$ be an algebra of type $(2,0)$. $\mathcal{A}$ is called a weak I-algebra if it satisfies

$$
\begin{aligned}
1 x & =x, \\
x x & =1, \\
x(y x) & =1, \\
(x y) y & =(y x) x
\end{aligned}
$$

and

$$
((x y) y) z=1 \quad \text { implies } \quad x z=1 .
$$

Remark 2.6. (S5) and (S1) imply (W5).

Theorem 2.7. Within the definition of a weak I-algebra (W3) and (W5) may be replaced by the laws

$$
((x y) y) y=x y
$$

and

$$
x((((x y) y) z) z)=1,
$$

respectively, and hence weak I-algebras form a variety.

Proof. (W4), (W3) and (W1) imply (W3'):

$$
((x y) y) y=(y(x y))(x y)=1(x y)=x y .
$$

(W2) and (W5) imply (W5'):

$$
((((x y) y) z) z)((((x y) y) z) z)=1
$$

and hence

$$
((x y) y)((((x y) y) z) z)=1
$$


whence

$$
x((((x y) y) z) z)=1 .
$$

(W3'), (W4) and (W2) imply (W3):

$$
x(y x)=((x(y x))(y x))(y x)=(((y x) x) x)(y x)=(y x)(y x)=1 .
$$

Finally, (W1) and (W5') imply (W5):

$$
((x y) y) z=1 \quad \text { implies } \quad x z=x(1 z)=x((((x y) y) z) z)=1 \text {. }
$$

Remark 2.8. The variety of weak $I$-algebras was characterized by the axioms (W1), (W2), (W3'), (W4) and (W5') in [3] where weak $I$-algebras were called $d$-implication algebras.

For the next theorem we need some definitions.

Definition 2.9. An algebra $(A, \sqcup)$ of type (2) is called a directoid (cf. [10]) if there exists a partial order relation $\leq$ on $A$ such that for all $a, b \in A, a \sqcup b$ is an upper bound of $a$ and $b$ that coincides with $\max (a, b)$ if $a$ and $b$ are comparable. $\leq$ is uniquely determined by $\sqcup$ by $x \leq y$ if and only if $x \sqcup y=y(x, y \in A)$. $(A, \sqcup)$ is called commutative if $\sqcup$ is commutative. Let $(P, \leq)$ be a poset with smallest element 0 and greatest element 1 and $f: P \rightarrow P . f$ is called a switching involution of $(P, \leq)$ if $f(0)=1, f(1)=0$ and $f(f(x))=x$ or all $x \in P$.

Now we can prove

Theorem 2.10. Let $\mathcal{A}=(A, \cdot, 1)$ be a weak I-algebra. Define

$$
x \sqcup y:=(x y) y \quad \text { and } \quad x^{y}:=x y
$$

for all $x, y \in A$. Then $\mathbf{S}(\mathcal{A}):=\left(A, \sqcup,\left({ }^{x} ; x \in A\right), 1\right)$ is an algebra such that $(A, \sqcup, 1)$ is a commutative directoid with greatest element 1 and for every $x \in A\left([x, 1], \leq{ }^{x}\right)$ is a poset with a switching involution where $\leq$ denotes the partial order induced by $\sqcup$. Conversely, let $\mathcal{S}:=\left(S, \sqcup,\left({ }^{x} ; x \in S\right), 1\right)$ be an algebra such that $(S, \sqcup, 1)$ is a commutative directoid with greatest element 1 and for every $x \in S$ $\left([x, 1], \leq,{ }^{x}\right)$ is a poset with a switching involution with respect to the partial order induced by $\sqcup$. Define

$$
x y:=(x \sqcup y)^{y}
$$

for all $x, y \in S$. Then $\mathbf{A}(\mathcal{S}):=(S, \cdot, 1)$ is a weak I-algebra. Moreover, $\mathbf{A}(\mathbf{S}(\mathcal{A}))=\mathcal{A}$ and $\mathbf{S}(\mathbf{A}(\mathcal{S}))=\mathcal{S}$ for every weak I-algebra $\mathcal{A}$ and every algebra $\mathcal{S}=\left(S, \sqcup,\left({ }^{x} ; x \in S\right), 1\right)$ such that $(S, \sqcup, 1)$ is a commutative directoid with greatest element 1 and for every $x \in S$ $\left([x, 1], \leq,^{x}\right)$ is a poset with a switching involution. 
Proof. First assume $\mathcal{A}=(A, \cdot, 1)$ to be a weak $I$-algebra and for all $x, y \in A$ define $x \leq y$ if $x y=1, x \sqcup y:=(x y) y$ and $x^{y}:=x y$. Reflexivity and antisymmetry of $\leq$ follow as in the proof of Theorem 2.4. If $a \leq b \leq c$ then $((a b) b) c=(1 b) c=b c=1$ according to (W1) whence $a c=1$ by (W5), i.e., $a \leq c$. This shows transitivity of $\leq$. Hence $(A, \leq)$ is a poset. That 1 is the greatest element of $(A, \leq)$ and $\sqcup$ is commutative follows as in the proof of Theorem 2.4. Because of (W2) we have $((a b) b)((a b) b)=1$ whence by (W5) it follows $a((a b) b)=1$, i.e., $a \leq$ $a \sqcup b$. Hence $b \leq b \sqcup a=a \sqcup b$. If $a \leq b$ then $a \sqcup b=(a b) b=1 b=b$ according to (W1). This shows that $(A, \sqcup, 1)$ is a commutative directoid with 1 . That for all $a \in A,{ }^{a}$ is an involution of $[a, 1]$ follows as in the proof of Theorem 2.4. Finally, $a^{a}=a a=1$ according to (W2) and $1^{a}=1 a=a$ according to (W1) showing that ${ }^{a}$ is switching. Therefore $\left.\mathbf{S}(\mathcal{A})=\left(A, \sqcup,{ }^{a} ; a \in A\right), 1\right)$ is an algebra such that $(S, \sqcup, 1)$ is a commutative directoid with greatest element 1 and for every $x \in S([x, 1]$, $\left.\leq,{ }^{x}\right)$ is a poset with a switching involution. Moreover, $((a b) b) b=a b$ follows as in the proof of Theorem 2.4 showing that $\mathbf{A}(\mathbf{S}(\mathcal{A}))=\mathcal{A}$.

Conversely, assume $\mathcal{S}=\left(A, \sqcup,\left({ }^{a} ; a \in A\right), 1\right)$ to be an algebra such that $(S, \sqcup, 1)$ is a commutative directoid with greatest element 1 and for every $x \in S\left([x, 1], \leq{ }^{x}\right)$ is a poset with a switching involution. Moreover, for all $x, y \in A$ define $x y:=(x \sqcup y)^{y}$.

(W1)-(W4) follow as in the proof of Theorem 2.4.

(W5) If $((a b) b) c=1$ then

$$
\begin{aligned}
& a \leq a \sqcup b \leq(a \sqcup b) \sqcup c=\left(\left(\left((a \sqcup b)^{b}\right)^{b} \sqcup c\right)^{c}\right)^{c} \\
& \quad=\left(\left(\left((a \sqcup b)^{b} \sqcup b\right)^{b} \sqcup c\right)^{c}\right)^{c}=(((a b) b) c)^{c}=1^{c}=c,
\end{aligned}
$$

which implies $a c=(a \sqcup c)^{c}=c^{c}=1$. Therefore $\mathbf{A}(\mathcal{S})=(A, \cdot, 1)$ is a weak $I$-algebra. $\left((a \sqcup b)^{b} \sqcup b\right)^{b}=a \sqcup b$ follows as in the proof of Theorem 2.4 and, moreover, $a \leq b$ implies $(b \sqcup a)^{a}=b^{a}$ showing that $\mathbf{S}(\mathbf{A}(\mathcal{S}))=\mathcal{S}$.

Remark 2.11. In [3] commutative directoids with greatest element 1 such that for every $x \in S\left([x, 1], \leq,{ }^{x}\right)$ is a poset with a switching involution were called commutative directoids with sectional antitone involutions.

\section{Congruence Kernels}

The aim of this section it to characterize congruence kernels of weak $I$-algebras having certain additional properties. First we observe that weak $I$-algebras are weakly regular which means that congruences are determined by the class of 1 :

Lemma 3.1. Let $\mathcal{A}=(A, \cdot, 1)$ be a weak I-algebra and $\Theta \in \operatorname{Con} \mathcal{A}$. Then $\Theta=\left\{(x, y) \in A^{2} \mid x y, y x \in[1] \Theta\right\}$. 
Proof. If for $a, b \in A$ it holds $a \Theta b$ then $a b \Theta a a=1$ and $b a \Theta a a=$ 1 and if, conversely, $a b \Theta 1$ and $b a \Theta 1$ then $a=1 a \Theta(b a) a=$ $(a b) b \Theta 1 b=b$.

Next we define the notion of a congruence kernel of a weak $I$-algebra:

Definition 3.2. A subset $K$ of the base set $A$ of a weak $I$-algebra $\mathcal{A}$ is called a congruence kernel of $\mathcal{A}$ if there exists a congruence $\Theta \in \operatorname{Con} \mathcal{A}$ with $[1] \Theta=K$. Let $\operatorname{Ker} \mathcal{A}$ denote the set of all congruence kernels of $\mathcal{A}$.

Theorem 3.3. The mappings $\Theta \mapsto[1] \Theta$ and $K \mapsto\left\{(x, y) \in A^{2} \mid x y\right.$, $y x \in K\}$ are mutually inverse isomorphisms between $(\operatorname{Con} \mathcal{A}, \subseteq)$ and $(\operatorname{Ker} \mathcal{A}, \subseteq)$ and hence the latter is a complete lattice.

Proof. The proof follows immediately from Lemma 3.1.

Certain subsets of weak $I$-algebras have a nice property which will be used in the proof of the final theorem of this section. In the following for a subset $K$ of a weak $I$-algebra $(A, \cdot, 1)$ and an element $a$ of $A$ define $K a:=\{k a \mid k \in K\}$. More generally, for subsets $K, L$ of $A$ we define $K L:=\{k l \mid k \in K, l \in L\}$.

Lemma 3.4. Let $\mathcal{A}=(A, \cdot, 1)$ be a weak I-algebra, $K$ a subset of $A$, $a \in K$ and $b \in A$ and assume $a b \in K$ and $(K(K x)) x \subseteq K$ for all $x \in A$. Then $b \in K$.

Proof. $b=1 b=(a((b a) a)) b=(a((a b) b)) b \in(K(K b)) b \subseteq K$.

Now we can state and prove the characterization of congruence kernels of certain weak $I$-algebras:

Theorem 3.5. Let $\mathcal{A}=(A, \cdot, 1)$ be a weak I-algebra satisfying

$$
x(y z)=(x y)(x z) \quad \text { and } \quad(x y)((y z)(x z))=1
$$

and let $K$ be a subset of $A$. Then $K$ is a congruence kernel of $\mathcal{A}$ if and only if $1 \in K, A K \subseteq K$ and $(K(K x)) x \subseteq K$ for all $x \in A$.

Proof. Let $a, b, c \in A$ and $d, e \in K$.

First assume $K \in \operatorname{Ker} \mathcal{A}$. Then there exists a $\Theta \in \operatorname{Con} \mathcal{A}$ with $[1] \Theta=K$ and hence

$$
\begin{aligned}
1 \in[1] \Theta & =K, \\
a d \in[a 1] \Theta & =[a(a a)] \Theta=[1] \Theta=K
\end{aligned}
$$

and

$$
(d(e a)) a \in[(1(1 a)) a] \Theta=[(1 a) a] \Theta=[a a] \Theta=[1] \Theta=K
$$

proving $1 \in K, A K \subseteq K$ and $(K(K x)) x \subseteq K$ for all $x \in A$. 
Conversely, assume $1 \in K, A K \subseteq K$ and $(K(K x)) x \subseteq K$ for all $x \in A$. Put $\Phi:=\left\{(x, y) \in A^{2} \mid x y, y x \in K\right\}$.

Since $1 \in K, \Phi$ is reflexive.

Obviously, $\Phi$ is symmetric.

If $a \Phi b \Phi c$ then $a b, b a, b c, c b \in K$ and hence $a(b c) \in A K \subseteq K$ and $(a(b c))(a c)=((a b)(a c))(a c)=(1((a b)(a c)))(a c) \in(K(K(a c)))(a c) \subseteq$ $K$ whence $a c \in K$ according to Lemma 3.4. Interchanging the roles of $a$ and $c$ yields $c a \in K$ and hence $a \Phi c$. This shows transitivity of $\Phi$.

If $a \Phi b$ then $a b, b a \in K$ and hence $(a b)((b c)(a c))=1 \in K$ whence $(b c)(a c) \in K$ according to Lemma 3.4. Interchanging the roles of $a$ and $b$ yields $(a c)(b c) \in K$ and hence $a c \Phi b c$. This shows that $\Phi$ is a right congruence on $\mathcal{A}$.

If, finally, $a \Phi b$ then $a b, b a \in K$ and hence $(c a)(c b)=c(a b) \in$ $A K \subseteq K$. Interchanging the roles of $a$ and $b$ yields $(c b)(c a) \in K$ and hence $c a \Phi c b$. This shows that $\Phi$ is a left congruence on $\mathcal{A}$.

Altogether we have proved $\Phi \in \operatorname{Con} \mathcal{A}$. Since, obviously, $[1] \Phi=K$, the proof of the theorem is complete.

\section{Varieties of Implication Algebras}

In this section we prove that the different varieties of implication algebras mentioned within the paper form a strictly increasing chain with respect to inclusion.

Definition 4.1. Let $V_{1}, V_{2}, V_{3}, V_{4}$ and $V_{5}$ denote the variety of all implication algebras, orthoimplication algebras, orthomodular implication algebras, strong $I$-algebras and weak $I$-algebras, respectively.

Theorem 4.2. $V_{1} \subset V_{2} \subset V_{3} \subset V_{4} \subset V_{5}$.

Proof. The orthoimplication algebra corresponding to $\mathcal{M O}_{2}:=\mathbf{2}^{2}+$ $\mathbf{2}^{2}$ belongs to $V_{2} \backslash V_{1}$ since $[0,1]$ is not a Boolean algebra. (Here + denotes the horizontal sum.) The orthomodular implication algebra corresponding to $\mathcal{M O}_{2} \times \mathbf{2}^{1}$ with the Hasse diagram

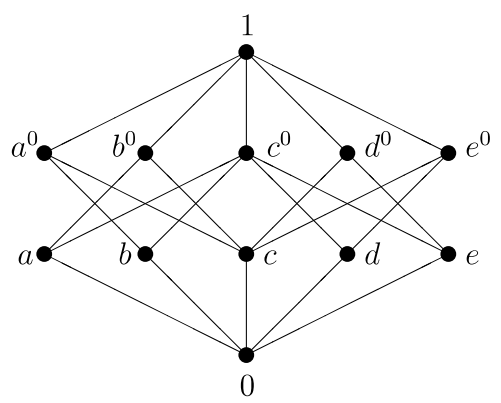


and with $\left(a^{0}\right)^{c}:=d^{0}$ and $\left(b^{0}\right)^{c}:=e^{0}$ belongs to $V_{3} \backslash V_{2}$ since $\left(a^{0}\right)^{c}=$ $d^{0} \neq b^{0}=a \vee c=\left(a^{0}\right)^{0} \vee c$. The strong $I$-algebra corresponding to the three-element chain belongs to $V_{4} \backslash V_{3}$ since $[0,1]$ is not an orthomodular lattice. The weak $I$-algebra corresponding to the poset with the Hasse diagram

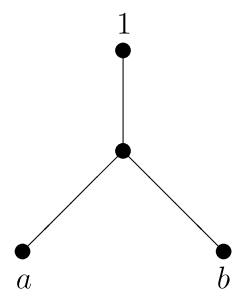

with $a \sqcup b:=1$ belongs to $V_{5} \backslash V_{4}$ since it is not a join-semilattice.

\section{References}

[1] Аввотт, J. C. (1967) Semi-boolean algebra. Mat. Vesnik 4: 177-198

[2] Аввотт, J. C. (1976) Orthoimplication algebras. Studia Logica 35: 173-177

[3] ChaJdA, I. (2007) Commutative directoids with sectional involutions. Discussiones Math. 27: 49-58

[4] Chajda, I. (2007) Ring-like structures derived from $\lambda$-lattices with antitone involutions. Math. Bohemica 132: 87-96

[5] Chajda, I., Eigenthaler, G. (2007) Semilattices with sectional mappings. Discussiones Math. 27: 11-19

[6] Chajda, I., EMAnovskÝ, P. (2004) Bounded lattices with antitone involutions and properties of MV-algebras. Discussiones Math. 24: 31-42

[7] Chajda, I., Halaš, R., KüHR, J. (2005) Distributive lattices with sectionally antitone involutions. Acta Sci. Math. (Szeged) 71: 19-33

[8] ChAJDA, I., HALAŠ, R., LÄNGER, H. (2001) Orthomodular implication algebras. Intern. J. Theor. Phys. 40: 1875-1884

[9] ChajdA, I., Halaš, R., LÄNGER, H. (2004) Simple axioms for orthomodular implication algebras. Intern. J. Theor. Phys. 43: 911-914

[10] JeŽEK, J., QuACKenbush, R. (1990) Directoids: Algebraic models of updirected sets. Algebra Universalis 27: 49-69

Authors' addresses: Ivan Chajda, Department of Algebra and Geometry, Palacký University Olomouc, Tomkova 40, 77900 Olomouc, Czech Republic. E-Mail: chajda@inf.upol.cz; Helmut Länger, Institute of Discrete Mathematics and Geometry, Vienna University of Technology, Wiedner Hauptstraße 8-10, 1040 Vienna, Austria. E-Mail: h.laenger@tuwien.ac.at. 